\title{
Should a standard lymphadenectomy during pancreatoduodenectomy exclude para-aortic lymph nodes for all cases of resectable pancreatic head cancer? A consensus statement by the Chinese Study Group for Pancreatic Cancer (CSPAC)
}

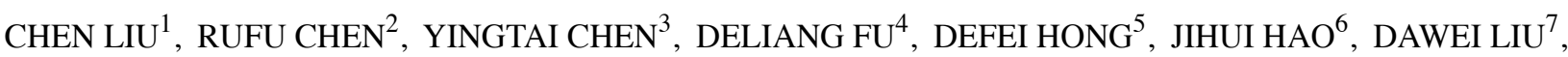 \\ JIANGTAO LI ${ }^{8}$, SHENGPING LI ${ }^{9}$, YIXIONG LI ${ }^{10}$, GANG MAI $^{11}$, YIPING MOU ${ }^{12}$, QUANXING NI ${ }^{1}$, \\ LI PENG $^{13}$, HONGGANG QIAN ${ }^{14}$, RENYI QIN ${ }^{15}$, BEI SUN ${ }^{16}$, CHENGHAO SHAO $^{17}$, YONGWEI SUN ${ }^{18}$, \\ BOLE TIAN $^{19}$, JIAN WANG ${ }^{18}$, WEI WANG ${ }^{20}$, WEILIN WANG ${ }^{21}$, GANG ZHAO $^{22}$ and XIANJUN YU ${ }^{1}$, \\ FOR THE CHINESE STUDY GROUP FOR PANCREATIC CANCER (CSPAC)
}

${ }^{1}$ Department of Pancreatic and Hepatobiliary Surgery, Fudan University Shanghai Cancer Center; Department of Oncology,

Shanghai Medical College, Fudan University; Pancreatic Cancer Institute, Fudan University, Shanghai;

${ }^{2}$ Department of Pancreaticobiliary Surgery, Sun Yat-sen Memorial Hospital, Sun Yat-sen University, Guangzhou;

${ }^{3}$ Department of Abdominal Surgery, Cancer Hospital, Chinese Academy of Medical Sciences, Peking Union Medical College,

Beijing; ${ }^{4}$ Department of Pancreatic Surgery, Pancreatic Disease Institute, Huashan Hospital, Shanghai Medical College,

Fudan University, Shanghai; ${ }^{5}$ Department of Hepatobiliary and Pancreatic Surgery, Zhejiang Provincial People's Hospital,

Hangzhou; ${ }^{6}$ Department of Pancreatic Cancer, Tianjin Medical University Cancer Institute and Hospital, Tianjin;

${ }^{7}$ Department of General Surgery of Heilongjiang Provincial Hospital, Harbin, Heilongjiang; ${ }^{8}$ Department of Surgery, Second Affiliated Hospital of Zhejiang University School of Medicine, Hangzhou; ${ }^{9}$ Department of Hepatobiliary Oncology,

Cancer Center, Sun Yat-sen University, Guangzhou; ${ }^{10}$ Department of Pancreatic-Bililary Surgery, Xiangya Hospital,

Central South University, Changsha, Hunan; ${ }^{11}$ Department of Hepatobiliopancreatic Surgery, The People's Hospital of Deyang,

Deyang; ${ }^{12}$ Department of Gastroenterological and Pancreatic Surgery, Zhejiang Provincial People's Hospital, Hangzhou;

${ }^{13}$ Department of Hepato-Pancreato-Biliary Surgery, The Fourth Hospital of Hebei Medical University, Shijiazhuang;

${ }^{14}$ Department of Hepato-Pancreato-Biliary Surgery, Peking University Cancer Hospital and Institute, Beijing;

${ }^{15}$ Department of Biliary-Pancreatic Surgery, Affiliated Tongji Hospital, Tongji Medical College,

Huazhong University of Science and Technology, Wuhan; ${ }^{16}$ Department of Pancreatic and Biliary Surgery,

The First Affiliated Hospital of Harbin Medical University, Harbin, Heilongjiang; ${ }^{17}$ Department of Pancreatic-Biliary Surgery,

Shanghai Changzheng Hospital, Second Military Medical University; ${ }^{18}$ Department of Biliary-Pancreatic Surgery,

Renji Hospital, School of Medicine, Shanghai Jiaotong University, Shanghai; ${ }^{19}$ Department of Hepato-Biliary-Pancreatic Surgery, West China Hospital, Sichuan University, Chengdu; ${ }^{20}$ Department of Surgery, Huadong Hospital, Fudan University, Shanghai;

${ }^{21}$ Department of Hepatobiliary and Pancreatic Surgery, First Affiliated Hospital, Zhejiang University School of Medicine, Hangzhou; ${ }^{22}$ The Pancreatic Disease Institute, Union Hospital, Tongji Medical College,

Huazhong University of Science and Technology, Wuhan, P.R. China

Received July 29, 2015; Accepted August 10, 2015

DOI: $10.3892 /$ ijo.2015.3128

\begin{abstract}
Correspondence to: Dr Xianjun Yu, Department of Pancreatic and Hepatobiliary Surgery, Shanghai Cancer Center, Pancreatic Cancer Institute, Fudan University, 270 Dong An Road, Shanghai 200032, P.R. China

E-mail: yuxianjun@fudanpci.org
\end{abstract}

Key words: pancreatic cancer, para-aortic lymph nodes, metastasis, extended lymphadenectomy, pancreatoduodenectomy
Abstract. Understanding and formulating an appropriate strategy for the para-aortic lymph nodes (LN16) during curative surgery for pancreatic head cancer have been controversial for some time. This study intended to provide a recommendation for surgeons to perform an optimal curative surgery on pancreatic cancer patients with or without LN16 involvement. Based on an updated literature search and review, the members of the Chinese Study Group for Pancreatic Cancer (CSPAC) from high-volume centers reached a consensus on the issue of LN16 in pancreatic head cancer. Metastasis to LN16 is quite 
common in pancreatic head cancer cases. Depending on the location of the tumor, including the ventral and dorsal pancreas, there could be various lymph node drainage pathways whereby LN16 does not necessarily belong to the Group 3 lymph node stations for all cases of pancreatic head cancer. Although LN16 involvement generally indicates a poor prognosis, some cohorts of LN16-involved cases have benefited from a curative surgery, and there is still a lack of level I evidence to convince surgeons to abandon all resectable cases with LN16 positivity. Resection of LN16 combined with a standard lymphadenectomy during pancreatoduodenectomy is recommended by CSPAC, except in patients with both positive LN16 and criteria based on: i) the resectability status of primary tumor; ii) the extent of involved para-aortic lymph nodes; and iii) the serum tumor burden assessed preoperatively.

\section{Introduction}

Pancreatic cancer, a biologically aggressive tumor with early metastasis and a dismal prognosis, has increased in global incidence in recent decades and is a major cause of death worldwide $(1,2)$. However, most patients are locally advanced or metastatic cases when diagnosed and have to rely on chemotherapy, radiation or even palliative care (3). Less than $20 \%$ of patients are suitable for a radical surgical procedure; therefore, it is critical for surgeons to establish a standard surgical procedure that can be widely accepted, especially with regard to the optimal lymphadenectomy protocol during the resection (4-6).

As for determining the optimal lymphadenectomy during pancreatoduodenectomy for pancreatic head cancer, whether an extended lymphadenectomy should be performed during a radical resection remained controversial. However, a recent multicenter randomised controlled trial (RCT) with 244 enrolled cases showed that extended lymphadenectomy including the dissection of lymph node stations $8,9,12,13$, 14,16 and 17 did not result in a survival benefit for pancreatic head cancer patients compared with a standard procedure that only includes lymph nodes 12c, 13 and 17 (7). Likewise, a consensus statement from the ISGPS (International Study Group on Pancreatic Surgery) declared that a standard lymph node resection of $\mathrm{LN}$ stations 5, 6, 8a, 12b, 12c, 13, 14a, 14b and 17 should be recommended rather than an extended dissection (8). Within both authoritative reports, there still remained disputes regarding the dissection of LN stations 8, 12 and 14 as a standard procedure. However, it was recommended that the resection of para-aortic lymph nodes (LN16), which consistently fell into the category of an extended lymphadenectomy, not be routinely performed during Whipple surgery.

Based on the Japanese Pancreas Society (JPS) staging systems for pancreatic cancer, the para-aortic lymph nodes (LN16) were categorized into a Group 3 lymph node station, the involvement of which was generally regarded as indicative of distant metastasis for pancreatic head cancer (9). Nevertheless, the situation of LN16 positivity in pancreatic head cancer is relatively common. In some cases, it is even more common than the involvement of the Group 1 or 2 lymph node stations (10-12). Despite the lack of level I evidence, some cohorts of patients with metastatic LN16 could still benefit from surgery, and a multicenter study that included 822 cases even declared that metastasis to LN16 was not an independent prognostic factor for pancreatic cancer (13). Consequently, it still remains embarrassing for a surgeon to encounter a confirmed or suspected metastatic LN16 in surgical procedure with a resectable pancreatic cancer. We are left with the question of whether the curative resection should be abandoned regardless of whether the primary tumor is resectable, only because the positive para-aortic lymph nodes are pathologically confirmed.

To address the issue of whether para-aortic lymph nodes should be excluded from standard lymphadenectomy for all cases of pancreatic head cancer, an expert panel from high volume centers in China participated in a consensus conference hosted by the Chinese Study Group for Pancreatic Cancer (CSPAC) in April 2015 to review the published literature and discuss the indication of LN16 resection in a Whipple procedure for pancreatic cancer. This first Chinese expert consensus from the CSPAC on pancreatic cancer formulated a personalized proposal on the extent of lymph node dissection for different subgroups of resectable pancreatic head cancer, which may add to the armamentarium available to pancreatic surgeons.

\section{Materials and methods}

For the consensus statement, a PubMed literature search was performed in Feb 2015 by entering the terms including 'pancreatic cancer', 'pancreatic head cancer', 'carcinoma of the head of the pancreas', 'lymph node metastasis', 'extended lymphadenectomy', 'para-aortic lymph node', 'pancreatoduodenectomy', 'metastasis', 'dorsal pancreas', 'ventral pancreas', 'mesopancreas', 'total mesopancreas excision', 'R0 resection', 'R1 resection', 'prognosis' and 'lymph node sampling'. According to the evidence level recommended by the Oxford Centre for Evidence-based Medicine, the reports were evaluated and rated; all case reports and non-English papers were excluded. A draft of the consensus statement was prepared by two CSPAC members (CL and XJY), then discussed, followed by an agreement by CSPAC members at a conference held in April 2015 in Shanghai, China.

\section{Results and consensus statements}

i) The strategy for resectable pancreatic head cancer cases in which LN16 is not suspected to be involved by pre-or intra-operative judgment. Although the LN16 nodes were defined as Group 3 lymph node stations by JPS, the involvement of para-aortic lymph nodes (LN16) was quite common in pancreatic head cancer cases. Nakao et al reported that the incidence of LN16 positivity in pancreatic head cancer was as high as $26 \%$ (12). Several other studies also found a high rate of LN16 metastasis that was close to or even higher than the incidence of some Group 2 lymph node stations such as LN8, LN12 (10-12,14). In a more recent study, metastasis to LN16 was surprisingly found to be as common as Group 1 lymph node stations (15). A Japanese study of the lymphatic drainage pathway for the pancreatic head area also inferred that the lymph node stations around the superior mesenteric artery (SMA) that belong to Group 2 were not necessarily superior to LN16 stations (16). Based on embryonic development, the head of the pancreas can be anatomically divided into two segments, the ventral and dorsal pancreas (17). Accordingly, 


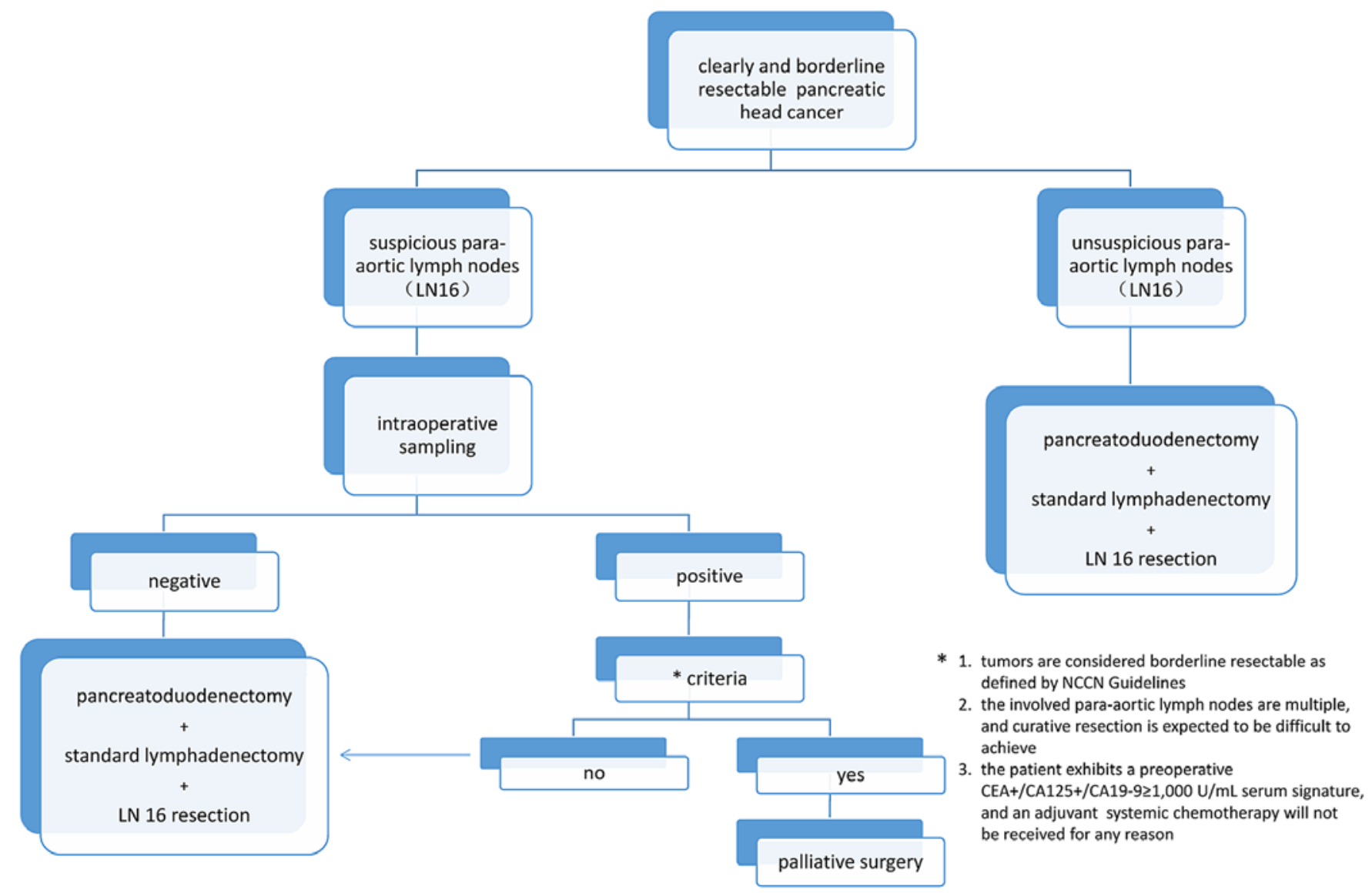

Figure 1. The strategy for resectable pancreatic head cancer cases where involvement of LN16 is suspected, or not suspected.

we wondered if there is any possibility that there are two or more different patterns of lymphatic metastasis for pancreatic head cancer. Although several studies in Asian centers have confirmed that there are different lymphatic flow characteristics for pancreatic head cancer between the ventral and dorsal pancreas $(18,19)$, level I evidence is still lacking to demonstrate that para-aortic lymph nodes should belong to some group of lymph node stations that are superior to Group 3 in certain parts of the head of the pancreas based on tumor location.

For pancreatic head ductal carcinoma, achieving a R0 resection, which is widely acknowledged as a critical prognostic factor for this disease, or minimizing the rate of R1 resection is the ultimate goal for a pancreatic surgeon $(7,20,21)$. Consequently, avoiding the involvement of a retroperitoneal margin in pancreatic surgery, and an en bloc resection of specimen including the primary tumor, peripancreatic lymph nodes, and mesopancreas becomes increasingly appreciated and commended by experts from several high volume centers (21-24). The mesopancreas is a virtual anatomic concept that consists of soft tissues, lymph nodes, vessels, and nerve fibers between the uncinate process of the pancreas and SMA, which is anatomically continuous with the para-aortic area. During the pancreaticoduodenectomy, a total mesopancreas excision will be smoothly performed when an extensive Kocher's maneuver has been accomplished, and then para-aortic lymph nodes are easily incorporated into the resection plane $(24,25)$. We questioned whether a surgeon should abandon the resection of a group of lymph nodes that will be easily removed and subsequently $\mathrm{R} 0$ resection could be achieved, just because these lymph nodes are probably imprecisely categorized into Group 3 stations.

Consensus statements. There is a relatively high incidence of LN16 involvement in pancreatic head ductal carcinoma, along with uncertainty about the precise lymphatic drainage pathway and category of para-aortic lymph nodes stations in the dorsal, ventral or both locations of the head of the pancreas. Therefore, in cases where pre-operative imaging signs or intra-operative detection suggest that LN16 is not involved, surgeons are suggested to dissect the para-aortic lymph nodes including 16a 2 and 16b1, which should be easily incorporated into a resection plane when an excision with curative intent has performed in addition to a standard lymphadenectomy (Fig. 1).

ii) The strategy for resectable pancreatic head cancer cases in which pre-or intra-operative judgments suspect LN16 involvement. Indeed, for pancreatic head ductal carcinoma, metastasis to LN16 is perceived as a factor of poor prognosis, as demonstrated by most reports $(15,26)$. Based on these reports, a pancreatic cancer patient with LN16 positivity is expected to live $<1$ year, and disease-free survival (DFS) is also estimated to be only $\sim 6$ months, even in cases who received curative resection. These survival rates are basically the same as those for cases who received chemotherapy or palliative care (26-28). However, a recent multicenter study of 822 cases reported that 
metastasis to para-aortic lymph nodes had no significant value in predicting survival (13), in addition, the presence of micrometastasis to LN16 was also found to have no affect on overall survival (OS) and DFS (27-29). As reported previously, there could exist some unique cohorts of patients with pancreatic carcinomas that cannot achieve any survival benefit from a surgery with curative intent. For instance, a preoperative high metabolic tumor burden measured by ${ }^{18} \mathrm{~F}-\mathrm{FDGPET} / \mathrm{CT}$ was significantly correlated with poor OS and recurrence-free survival (RFS) of resected pancreatic cancer cases (30), and a subsequent sizeable study demonstrated that a preoperative $\mathrm{CEA}^{+} / \mathrm{CA}_{125} / \mathrm{CA} 19-9 \geq 1,000 \mathrm{U} / \mathrm{ml}$ serum signature in resected pancreatic cancer patients can distinguish a subgroup of patients with dismal survival outcomes (31). Despite a similar probability of poor surgical outcome, some subgroups of LN16 positive cases have been shown to achieve long-term survival instead of an overall poor prognosis (32). With or without adjuvant or neoadjuvant chemotherapy, the numbers of involved LN16, primary tumor size, the age of the patient, and the presence of pathological portal vein invasion were all considered as critical impact factors that altered the prognosis after a curative resection of pancreatic cancer, even with LN16 metastasis $(13,15,32-34)$. A recent prospective study claimed the presence of LN16 involvement should be regarded as a contra-indication to curative resection for pancreatic cancer (28); however, due to lack of a subgroup analysis, level I evidence on the significance of metastasis to para-aortic lymph nodes in pancreatic head carcinoma still remains elusive. Thus, should the single indicator of LN16 positivity alone determine the destiny of patients to be provided or deprived of an opportunity to access a curative treatment?

It was reported that an intraoperative pathological sampling of para-aortic lymph nodes was necessary before a curative resection will be performed, and sensitivity and specificity to diagnose the involvements of LN16 by frozen sections was 70 and $100 \%$, respectively $(28,35)$. Para-aortic lymph nodes, which are supposed to be submitted for a frozen section assessment intraoperatively, reside between the celiac axis and the inferior mesenteric artery and are divided into $16 \mathrm{a} 2$ and $16 \mathrm{~b} 1$ by the left renal vein $(28,35)$. In the light of its retroperitoneal location, it is technically difficult to acquire histopathological evidence by sampling that can completely reveal the profile of the para-aortic lymph nodes as involved, 'micro-involved' or not involved. Although several promising techniques such as intra-operative ultrasound localization and a transperitoneal laparoscopic approach were introduced into clinical practice (36), it is still uncommon for LN16 to be sampled before performing a curative resection for all the resectable pancreatic cancer cases due to an issue of sensitivity.

Consensus statements. According to the aforementioned facts, a careful intraoperative sampling is recommended before a curative intent surgery is performed only when para-aortic lymph nodes are highly suspicious to be involved as judged pre- or intra-operatively by qualified surgeons. If the histopathological results are positive, a palliative surgery such as by-pass procedure will be performed, provided that the patients meet any of the following criteria: i) tumors are considered borderline resectable as defined by NCCN
Guidelines (version 1.2014); ii) the metastatic para-aortic lymph nodes are multiple, and curative resection is expected to be difficult to achieve; and iii) the patient exhibits a preoperative $\mathrm{CEA}^{+} / \mathrm{CA} 125^{+} / \mathrm{CA} 19-9 \geq 1,000 \mathrm{U} / \mathrm{ml}$ serum signature, and an adjuvant systemic chemotherapy will not be received for any reason. Otherwise, a curative surgery combined with LN16 resection is still recommended (Fig. 1).

\section{Discussion}

Unlike other digestive organs such as the stomach, small intestine, and colon, the pancreas does not have a real anatomic mesopancreas that usually accompanies the peripancreas lymphatic drainage pathway. Particularly, the head of pancreas consists of two parts of embryological segments of which ventral pancreas is bound to dorsal pancreas by a connecting line between common bile duct and superior mesenteric vein (17). Due to the close adjacency to the main venous system, including the renal vein and inferior vena cava, LN16 involvement could develop into a systemic metastasis in early disease stage even if LN16 should have been categorized as belonging to a proximal lymph node station rather than a distant station. This possibility is especially likely for a primary tumor in the dorsal pancreas such as the uncinate process. Accordingly, the lymphatic drainage pathway in pancreatic head ductal carcinoma could be more complicated depending on tumor location, instead of a single grouping of lymph node stations for every single case.

Pancreatic cancer is a biologically unique tumor, and there could be one or more cohorts of patients who cannot benefit from all available therapeutic regimens even with a radical resection (31). Although the involvement of a para-aortic lymph node is indicated as a poor prognostic factor by most studies $(26,28,32,33)$, level I evidence or sizeable, multiple center, prospective, controlled trials, especially those with a subgroup analysis of LN16 status based on different tumor locations for pancreatic head cancer, still appear insufficient. Several retrospective studies declared that not all the cases with LN16 positivity always exhibit poor prognostic signatures $(13,15,32-34)$, and anatomically, the para-aortic area is continuous with the mesopancreas and LN16 can technically be incorporated into a surgical resection plane when $\mathrm{R} 0$ intent resection is performed $(24,25)$. Consequently, LN16 (16a2, 16b1) dissection is suggested to be routinely included in a standard lymphadenectomy during pancreatoduodenectomy for pancreatic head cancer by CSPAC, unless any contraindication mentioned above mentioned is present.

The current consensus concerning the issue of para-aortic lymph nodes in the setting of resectable pancreatic head cancer (including clearly and borderline resectable cases according to NCCN Guidelines version 1.2014) is supported and unanimously approved by CSPAC members from several high volume pancreas centers in China and suggests that an optimal lymphadenectomy should be performed during the Whipple procedure for experienced pancreatic surgeons in specialized centers. Although the dissection of para-aortic lymph nodes is recommended by the CSPAC in most cases, a multimodal therapy particularly including systemic chemotherapy should be underlined in the cases in which LN16 nodes are pathologically involved postoperatively. 


\section{References}

1. Li D, Xie K, Wolff R and Abbruzzese JL: Pancreatic cancer. Lancet 363: 1049-1057, 2004.

2. Shi S, Yao W, Xu J, Long J, Liu C and Yu X: Combinational therapy: New hope for pancreatic cancer? Cancer Lett 317 $127-135,2012$

3. Long J, Luo GP, Xiao ZW, Liu ZQ, Guo M, Liu L, Liu C, Xu J, Gao YT, Zheng Y, et al: Cancer statistics: Current diagnosis and treatment of pancreatic cancer in Shanghai, China. Cancer Lett 346: 273-277, 2014.

4. Liu C, Long J, Liu L, Xu J, Zhang B, Yu X and Ni Q: Pancreatic stump-closed pancreaticojejunostomy can be performed safely in normal soft pancreas cases. J Surg Res 172: e11-e17, 2012

5. Pedrazzoli S, DiCarlo V, Dionigi R, Mosca F, Pederzoli P, Pasquali C, Klöppel G, Dhaene K and Michelassi F; Lymphadenectomy Study Group: Standard versus extended lymphadenectomy associated with pancreatoduodenectomy in the surgical treatment of adenocarcinoma of the head of the pancreas: A multicenter, prospective, randomized study. Ann Surg 228: 508-517, 1998.

6. Farnell MB, Pearson RK, Sarr MG, DiMagno EP, Burgart LJ, Dahl TR, Foster N and Sargent DJ; Pancreas Cancer Working Group: A prospective randomized trial comparing standard pancreatoduodenectomy with pancreatoduodenectomy with extended lymphadenectomy in resectable pancreatic head adenocarcinoma. Surgery 138: 618-630, 2005.

7. Jang JY, Kang MJ, Heo JS, Choi SH, Choi DW, Park SJ, Han SS Yoon DS, Yu HC, Kang KJ, et al: A prospective randomized controlled study comparing outcomes of standard resection and extended resection, including dissection of the nerve plexus and various lymph nodes, in patients with pancreatic head cancer. Ann Surg 259: 656-664, 2014.

8. Tol JA, Gouma DJ, Bassi C, Dervenis C, Montorsi M, Adham M, Andrén-Sandberg A, Asbun HJ, Bockhorn M, Büchler MW, et al; International Study Group on Pancreatic Surgery: Definition of a standard lymphadenectomy in surgery for pancreatic ductal adenocarcinoma: A consensus statement by the International Study Group on Pancreatic Surgery (ISGPS). Surgery 156 591-600, 2014.

9. Kondo S: Japanese Pancreas Society staging systems for pancreatic cancer. In: Pancreatic Cancer. Neoptolemos JP, Urrutia R, Abbruzzese JL and Bücher MW (eds). Springer, New York, NY, pp1035-1050, 2010.

10. Kayahara M, Nagakawa T, Ueno K, Ohta T, Tsukioka Y and Miyazaki I: Surgical strategy for carcinoma of the pancreas head area based on clinicopathologic analysis of nodal involvement and plexus invasion. Surgery 117: 616-623, 1995.

11. Kayahara M, Nagakawa T, Kobayashi H, Mori K, Nakano T, Kadoya N, Ohta T, Ueno K and Miyazaki I: Lymphatic flow in carcinoma of the head of the pancreas. Cancer 70: 2061-2066, 1992.

12. Nakao A, Harada A, Nonami T, Kaneko T, Murakami H, Inoue $\mathrm{S}$, Takeuchi $\mathrm{Y}$ and Takagi $\mathrm{H}$ : Lymph node metastases in carcinoma of the head of the pancreas region. Br J Surg 82: 399-402, 1995.

13. Sho M, Murakami Y, Motoi F, Satoi S, Matsumoto I, Kawai M, Honda G, Uemura K, Yanagimoto H, Kurata M, et al: Postoperative prognosis of pancreatic cancer with para-aortic lymph node metastasis: A multicenter study on 822 patients. J Gastroenterol 50: 694-702, 2015.

14. Sakai M, Nakao A, Kaneko T, Takeda S, Inoue S, Kodera Y, Nomoto S, Kanazumi N and Sugimoto H: Para-aortic lymph node metastasis in carcinoma of the head of the pancreas. Surgery 137 606-611, 2005

15. Yamada S, Nakao A, Fujii T, Sugimoto H, Kanazumi N, Nomoto S, Kodera Y and Takeda S: Pancreatic cancer with paraaortic lymph node metastasis: A contraindication for radical surgery? Pancreas 38: e13-e17, 2009.

16. Hirono S, Tani M, Kawai M, Okada K, Miyazawa M, Shimizu A, Uchiyama $\mathrm{K}$ and Yamaue $\mathrm{H}$ : Identification of the lymphatic drainage pathways from the pancreatic head guided by indocyanine green fluorescence imaging during pancreaticoduodenectomy. Dig Surg 29: 132-139, 2012.

17. Okamura Y, Fujii T, Kanzaki A, Yamada S, Sugimoto H, Nomoto S, Takeda $\mathrm{S}$ and Nakao A: Clinicopathologic assessment of pancreatic ductal carcinoma located at the head of the pancreas, in relation to embryonic development. Pancreas 41 : $582-588,2012$
18. Makino I, Kitagawa $\mathrm{H}$, Ohta $\mathrm{T}$, Nakagawara $\mathrm{H}$, Tajima $\mathrm{H}$, Ohnishi I, Takamura H, Tani T and Kayahara M: Nerve plexus invasion in pancreatic cancer: Spread patterns on histopathologic and embryological analyses. Pancreas 37: 358-365, 2008.

19. Kitagawa H, Ohta T, Makino I, Tani T, Tajima H, Nakagawara H, Ohnishi I, Takamura $\mathrm{H}$, Kayahara M, Watanabe $\mathrm{H}$, et al: Carcinomas of the ventral and dorsal pancreas exhibit different patterns of lymphatic spread. Front Biosci 13: 2728-2735, 2008.

20. Hartwig W, Vollmer CM, Fingerhut A, Yeo CJ, Neoptolemos JP, Adham M, Andrén-Sandberg A, Asbun HJ, Bassi C, Bockhorn M, et al; International Study Group on Pancreatic Surgery: Extended pancreatectomy in pancreatic ductal adenocarcinoma: Definition and consensus of the International Study Group for Pancreatic Surgery (ISGPS). Surgery 156: 1-14, 2014.

21. Gaedcke J, Gunawan B, Grade M, Szöke R, Liersch T, Becker H and Ghadimi BM: The mesopancreas is the primary site for R1 resection in pancreatic head cancer: Relevance for clinical trials. Langenbecks Arch Surg 395: 451-458, 2010.

22. Gockel I, Domeyer M, Wolloscheck T, Konerding MA and Junginger T: Resection of the mesopancreas (RMP): A new surgical classification of a known anatomical space. World J Surg Oncol 5: 44, 2007.

23. Inoue Y, Saiura A, Yoshioka R, Ono Y, Takahashi M, Arita J, Takahashi Y and Koga R: Pancreatoduodenectomy with systematic Mesopancreas dissection using a Supracolic anterior artery-first approach. Ann Surg: Jan 13, 2015 (Epub ahead of print).

24. Adham M and Singhirunnusorn J: Surgical technique and results of total mesopancreas excision (TMpE) in pancreatic tumors. Eur J Surg Oncol 38: 340-345, 2012.

25. Peparini N and Chirletti P: Mesopancreas: A boundless structure, namely $\mathrm{R} 1$ risk in pancreaticoduodenectomy for pancreatic head carcinoma. Eur J Surg Oncol 39: 1303-1308, 2013.

26. Doi R, Kami K, Ito D, Fujimoto K, Kawaguchi Y, Wada M, Kogire M, Hosotani R, Imamura $\mathrm{M}$ and Uemoto S: Prognostic implication of para-aortic lymph node metastasis in resectable pancreatic cancer. World J Surg 31: 147-154, 2007.

27. Choi SH, Kim SH, Choi JJ, Kang CM, Hwang HK and Lee WJ: Clinical necessity of the immunohistochemical reassessment of para-aortic lymph nodes in resected pancreatic ductal adenocarcinoma. Oncol Lett 6: 1189-1194, 2013.

28. Schwarz L, Lupinacci RM, Svrcek M, Lesurtel M, Bubenheim M, Vuarnesson H, Balladur P and Paye F: Para-aortic lymph node sampling in pancreatic head adenocarcinoma. Br J Surg 101: 530-538, 2014 .

29. Kayahara M, Funaki K, Tajima H, Takamura H, Ninomiya I, Kitagawa $\mathrm{H}$ and Ohta T: Surgical implication of micrometastasis for pancreatic cancer. Pancreas 39: 884-888, 2010

30. Xu HX, Chen T, Wang WQ, Wu CT, Liu C, Long J, Xu J, Zhang YJ, Chen RH, Liu L, et al: Metabolic tumour burden assessed by ${ }^{18} \mathrm{~F}-\mathrm{FDG}$ PET/CT associated with serum CA19-9 predicts pancreatic cancer outcome after resection. Eur J Nucl Med Mol Imaging 41: 1093-1102, 2014.

31. Liu L, Xu H, Wang W, Wu C, Chen Y, Yang J, Cen P, Xu J, Liu C, Long J, et al: A preoperative serum signature of $\mathrm{CEA}^{+} / \mathrm{CA}^{2} 25^{+}$ CA19-9 $\geq 1000 \mathrm{U} / \mathrm{ml}$ indicates poor outcome to pancreatectomy for pancreatic cancer. Int J Cancer 136: 2216-2227, 2015.

32. Yamada S, Fujii T, Sugimoto H, Kanazumi N, Kasuya H, Nomoto S, Takeda S, Kodera Y and Nakao A: Pancreatic cancer with distant metastases: A contraindication for radical surgery? Hepatogastroenterology 56: 881-885, 2009.

33. Murakami Y, Uemura K, Sudo T, Hashimoto Y, Yuasa Y and Sueda T: Prognostic impact of para-aortic lymph node metastasis in pancreatic ductal adenocarcinoma. World J Surg 34: 1900-1907, 2010

34. Kanda M, Fujii T, Nagai S, Kodera Y, Kanzaki A, Sahin TT, Hayashi M, Yamada S, Sugimoto H, Nomoto S, et al: Pattern of lymph node metastasis spread in pancreatic cancer. Pancreas 40: 951-955, 2011.

35. Imai H, Doi R, Kanazawa H, Kamo N, Koizumi M, Masui T, Iwanaga Y, Kawaguchi Y, Takada Y, Isoda H, et al: Preoperative assessment of para-aortic lymph node metastasis in patients with pancreatic cancer. Int J Clin Oncol 15: 294-300, 2010

36. Kim H, Hyung WJ, Lim JS, Park MS, Choi JY, Chung YE, Kim MJ and Kim KW: Laparoscopic ultrasonography-assisted retroperitoneal lymph node sampling in patients evaluated for stomach cancer recurrence. J Ultrasound Med 27: 1229-1233, 2008. 